\title{
A Network Flow Approach to Predict Protein Targets and Flavonoid Backbones to Treat Respiratory Syncytial Virus Infection
}

\author{
José Eduardo Vargas, ${ }^{1}$ Renato Puga, ${ }^{2}$ Joice de Faria Poloni, ${ }^{3}$ \\ Luis Fernando Saraiva Macedo Timmers, ${ }^{4}$ Barbara Nery Porto, ${ }^{1}$ Osmar Norberto de Souza, \\ Diego Bonatto, ${ }^{3}$ Paulo Márcio Condessa Pitrez, ${ }^{1}$ and Renato Tetelbom Stein ${ }^{1}$ \\ ${ }^{1}$ Centro Infant, Pontifical Catholic University of Rio Grande do Sul (PUCRS), Avenue Ipiranga 6681, \\ 90619-900 Porto Alegre, RS, Brazil \\ ${ }^{2}$ Clinical Research Center, Hospital Israelita Albert Einstein (HIAE), São Paulo, Brazil \\ ${ }^{3}$ Department of Molecular Biology and Biotechnology, Federal University of Rio Grande do Sul (UFRGS), \\ 90619-900 Porto Alegre, RS, Brazil \\ ${ }^{4}$ Faculty of Informatics, Laboratory for Bioinformatics, Modelling \& Simulation of Biosystems, \\ Pontifical Catholic University of Rio Grande do Sul (PUCRS), 90619-900 Porto Alegre, RS, Brazil
}

Correspondence should be addressed to José Eduardo Vargas; josevargas123@gmail.com

Received 26 July 2014; Accepted 11 September 2014

Academic Editor: Jiangning Song

Copyright ( 2015 José Eduardo Vargas et al. This is an open access article distributed under the Creative Commons Attribution License, which permits unrestricted use, distribution, and reproduction in any medium, provided the original work is properly cited.

\begin{abstract}
Background. Respiratory syncytial virus (RSV) infection is the major cause of respiratory disease in lower respiratory tract in infants and young children. Attempts to develop effective vaccines or pharmacological treatments to inhibit RSV infection without undesired effects on human health have been unsuccessful. However, RSV infection has been reported to be affected by flavonoids. The mechanisms underlying viral inhibition induced by these compounds are largely unknown, making the development of new drugs difficult. Methods. To understand the mechanisms induced by flavonoids to inhibit RSV infection, a systems pharmacologybased study was performed using microarray data from primary culture of human bronchial cells infected by RSV, together with compound-proteomic interaction data available for Homo sapiens. Results. After an initial evaluation of 26 flavonoids, 5 compounds (resveratrol, quercetin, myricetin, apigenin, and tricetin) were identified through topological analysis of a major chemical-protein (CP) and protein-protein interacting (PPI) network. In a nonclustered form, these flavonoids regulate directly the activity of two protein bottlenecks involved in inflammation and apoptosis. Conclusions. Our findings may potentially help uncovering mechanisms of action of early RSV infection and provide chemical backbones and their protein targets in the difficult quest to develop new effective drugs.
\end{abstract}

\section{Introduction}

Respiratory syncytial virus (RSV) is a major cause of lower respiratory tract infection with high level of mortality in children around the world [1-3]. It is estimated that all children by two years of age have been infected by RSV and more than half of them are reinfected [4]. Moreover, RSV pathogenesis is notably associated with an increased airway resistance characterized as wheezing, diagnosed as bronchiolitis [2].

In the 1960 decade, a vaccine trial was performed with unexpected and tragic results [5]. Hence, effective preventive treatment to RSV infection is unavailable, since there is no vaccine against the virus. However, several prototypes are under study [6-9]. The prophylactic therapy with palivizumab, a humanized monoclonal antibody, has been 
shown to reduce the number of RSV hospitalizations in preterm infants [10], but the treatment has a very high cost, and it is administered only to children with risk factors for RSV bronchiolitis [11]. Another optional treatment against RVS infection is ribavirin. It is a nucleoside analog that introduces mutations into the RNA viral genome during replication and was previously used routinely for infants hospitalized with RSV. However, it has been associated with undesired side-effects and was not considered an effective treatment $[12,13]$.

The absence of a vaccine for RSV-induced bronchiolitis and the existence of few antiviral agents against RSV constitute very important problems in pediatric medicine. Thus, the development of novel anti-RSV drugs that can be administered orally or parenteral to children is extremely necessary.

A great variety of viruses have been reported to be inhibited by natural compounds, such as flavonoids [14-16]; however, the molecular mechanisms underlying such effects are largely unclear. In this sense, it is difficult to develop new drugs.

In a search to provide new insights for RSV treatments and to understand the multiples signaling pathways affected by RSV infection, an integrative model based on systems pharmacology predictions has been used. Moreover, this methodology will allow understanding the effect of flavonoid (FLA) compounds against RSV infection, integrating chemical-protein (CP) and protein-protein interaction (PPI) networks.

\section{Materials and Methods}

2.1. Gene Expression Data from Primary Human Bronchial Epithelial (PHBE) Cells Infected by RSV. The microarray data GSE12144 were downloaded from the Gene Expression Omnibus (GEO) database [http://www.ncbi.nlm.nih .gov/geo/]. Subsequently, a linear model was applied to normalize this data, using Limma package from R/Bioconductor to guarantee maximal statistical stringency [17]. Additionally, a contrast analysis was applied and differentially expressed genes (PHBE mock versus PHBE RSV 24h) were identified by Rank Product with a cutoff $P$ value of $\leq 0.05$ [18].

2.2. Selection of Flavonoids. To select flavonoids with potential antiviral effect against pathogenic respiratory agents, a literature mining was performed. Two flavonoids commonly described against respiratory viral infections were selected: quercetin [19-21] and resveratrol [22-24]. Quercetin is found in abundance in onions, apples, broccoli, and berries [25], whereas resveratrol is present in grapes, berries, and peanuts [25].

In order to obtain drug-like compounds, a databasedependent model was applied to calculate the drug-likeness of all compounds similar to resveratrol or quercetin through Tanimoto coefficient (Tc) [37]:

$$
\mathrm{Tc}=\frac{\sum_{j=1}^{k} a_{j} \times b_{j}}{\left(\sum_{j=1}^{k} a_{j}^{2}+\sum_{j=1}^{k} b_{j}^{2}-\sum_{j=1}^{k} a_{j} \times b_{j}\right)},
$$

where " $a$ " is the molecular property of each compound and " $b$ " represents the average molecular properties of the whole compounds in the Drugbank database [http://www.drugbank.ca/]. The Drugbank database is a unique bioinformatic resource that contains 6825 compound data. These chemical compounds are FDA approved drugs or are being evaluated in clinical trials. In our work a criterion of Tc values $\geq 0.611$ was used according to suggestion by Drugbank site (data shown in the Table 1).

2.3. Design of CP-PPI Networks. To obtain CP-PPI networks, the metasearch engine STITCH 3.1 [http://stitch.embl.de/] was applied. STITCH software allows visualization of the connections (edge) among different proteins, chemical compounds, and compounds-proteins, where each edge shows a degree of confidence between 0 (lowest confidence) and 1.0 (highest confidence). To this present work, the parameters used were as follows: all prediction methods were enabled, excluding text mining; maximal of 10 interactions by node; degree of confidence, medium (0.400); and a network depth equal to 1 . In addition, GeneCard [http://www.genecards.org/] and Pubchem [https://pubchem.ncbi.nlm.nih.gov/] databases were used to search synonymous names of genes and compounds recognizable by STITCH. In sequence, the outcomes obtained through these search engines were analyzed with Cytoscape 2.8.2 [38]. Nonconnected nodes were excluded from the networks.

2.4. Modular Analysis of CPI-PPI Network. ClusterONE was the tool used to discover densely connected and possibly overlapping regions within the Cytoscape network [39]. Dense regions corresponded to protein or compound-protein complexes or parts of them.

ClusterONE identifies subnetworks by the identification of "growing" dense regions out of small seeds guided by a quality function. The quality of a group was evaluated by the number of internal edges divided by the number of edges involving nodes of the group.

2.5. Gene Ontology Analysis. Gene ontology (GO) analysis was determined by biological network gene ontology (BiNGO) software 2.44 [http://chianti.ucsd.edu/cyto_web/ plugins/index.php] [40]. The degree of functional enrichment for a given category was assessed $(P$ value $\leq 0.05)$ by hypergeometric distribution [41] and multiple test correction was applied using the false discovery rate (FDR) algorithm [42], from BiNGO software. Overrepresented biological process categories were obtained after FDR correction, with a significance level of 0.05 .

2.6. Centralities Parameters and Topological Analysis. Major network centralities (closeness, betweenness, and node degree) were analyzed with the CP-PPI networks using the Cytoscape plugin CentiScape 2.8.2 [43]. 
TABLE 1: List of flavonoid compounds considered to chemical protein-protein network design. Chemical identification (Pubchem), Tanimoto similarity scores, and the antiviral activity of each compound (manually curated from literature).

\begin{tabular}{|c|c|c|c|}
\hline Compound ID & Pubchem CID & Tanimoto similarity (score) & Antiviral RSV references \\
\hline \multicolumn{4}{|l|}{$\mathbf{A}^{*}$} \\
\hline Resveratrol & 445154 & 1 & {$[22,24,26-29]$} \\
\hline Piceatannol & 667639 & 0.966 & $\mathrm{UD}^{* * *}$ \\
\hline $\mathrm{AC1O} 4 \mathrm{D} 7 \mathrm{M}$ & 6365297 & 0.719 & UD \\
\hline Caffeic acid & 689043 & 0.689 & UD \\
\hline Phenol & 996 & 0.687 & {$[30,31]$} \\
\hline HLF & 5288545 & 0.684 & UD \\
\hline Sinapinate & 637775 & 0.635 & UD \\
\hline Ferulic acid & 445858 & 0.622 & {$[32]$} \\
\hline Isoferulic acid & 736186 & 0.614 & {$[32]$} \\
\hline $2 \mathrm{MP}$ & 7249 & 0.621 & UD \\
\hline P-coumaric acid & 637542 & 0.611 & UD \\
\hline \multicolumn{4}{|l|}{$\mathbf{B}^{* *}$} \\
\hline Quercetin & 5280343 & 1 & {$[19,25]$} \\
\hline Myricetin & 5281672 & 1 & UD \\
\hline ST059620 & 5281614 & 0.959 & UD \\
\hline Kaempferol & 5280863 & 0.946 & {$[25]$} \\
\hline Tricetin & 5281701 & 0.884 & UD \\
\hline Apigenin & 5280443 & 0.823 & {$[33]$} \\
\hline Oroxylin A & 5320315 & 0.791 & {$[34]$} \\
\hline Wogonin & 5281703 & 0.765 & {$[34]$} \\
\hline Flavone & 10680 & 0.714 & {$[35]$} \\
\hline EMD 21388 & 128600 & 0.636 & UD \\
\hline$\alpha$-Naphthoflavone & 11790 & 0.711 & {$[34]$} \\
\hline$\beta$-Naphthoflavone & 2361 & 0.711 & {$[35]$} \\
\hline Rutin & 5280805 & 0.631 & UD \\
\hline Genistein & 5280961 & 0.618 & {$[36]$} \\
\hline DB07032 & 656936 & 0.612 & UD \\
\hline
\end{tabular}

$\mathrm{A}^{*}$ Group with high similarity to resveratrol. $\mathrm{B}^{* *}$ Group with high similarity to quercetin.

$\mathrm{UD}^{* * *}$ Undescribed in the literature.

Closeness centrality was used to evaluate the shortest path among a random node (protein or chemical compound) and all other nodes [43]:

$$
\operatorname{Clo}(v)=\frac{1}{\sum w \in v^{\operatorname{dist}(v, w)}}
$$

where the closeness value $(\mathrm{Clo}(v))$ was calculated by computing the shortest path between the node $v$ and all other nodes $w$ found within a network.

The average closeness (Clo) score was calculated by the sum of different closeness scores $\left(\mathrm{Clo}_{i}\right)$ divided by the total number of nodes analyzed $\left(N_{(v)}\right)$ :

$$
\langle\mathrm{Clo}\rangle=\frac{\sum_{i} \mathrm{Clo}_{i}}{N_{(v)}}
$$

Also, the betweenness parameter was taken into account in the analysis. This parameter is a measure equal to the number of shortest paths from a couple of nodes that pass through a different node $[43,44]$ :

$$
\operatorname{Bet}(v)=\sum_{s \neq v \neq w \in V} \frac{\sigma_{s w}(v)}{\sigma_{s w}}
$$

where $\sigma_{s w}$ is the total number of the shortest paths from node $s$ to node $w$ and $\sigma_{s w}(v)$ is the number of those paths that pass through the node $v$.

The average betweenness score (Bet) of the network was calculated using (5), where the sum of different betweenness scores $\left(\mathrm{Bet}_{i}\right)$ is divided by the total number of nodes $N_{(v)}$ analyzed:

$$
\langle\text { Bet }\rangle=\frac{\sum_{i} \text { Bet }_{i}}{N_{(v)}}
$$


The average betweenness score of CP-PPI network was used to obtain responsible nodes of the control of the flow of information in the network. These nodes are called bottlenecks (B) and show higher probability of connections of different modules or biological processes.

Finally, parameter degree was calculated. This parameter is a measure that indicates the number of adjacent nodes $\left(E_{i}\right)$ that are connected to a specific node $(v)$, according to

$$
\operatorname{Deg}(v)=\sum E_{i} .
$$

The average node degree of a network (Deg) is given by (7), where the sum of different node degree scores $\left(\right.$ Deg $\left._{i}\right)$ is divided by the total number of nodes $N_{(v)}$ present in the network:

$$
\langle\mathrm{Deg}\rangle=\frac{\sum_{i} \operatorname{Deg}_{i}}{N_{(v)}}
$$

Nodes with a high node degree score compared to the average are called hubs $(\mathrm{H})$ and are responsible for a central regulatory role in the cell.

In this work, $\mathrm{H}-\mathrm{B}$ (hub-bottleneck) may correspond to central proteins or FLA compounds that are highly connected to several complexes, while nodes that belong to the $\mathrm{NH}$ (non-hub-B) group correspond to proteins or FLA compounds that are important. In order to obtain $\mathrm{H}-\mathrm{B}$ and $\mathrm{NH}$-nodes, mathematical means (threshold) generated for betweenness and degree parameters were considered.

2.7. Molecular Parameters for the Development of a Potential Drug. All compounds, which were chemically verified by Zinc database $[45,46]$ were analyzed taking into account the Lipinsky's rule of five (xLogP, molecular weight, number of hydrogen bond acceptors, and donors). Toxicity risks (mutagenic, tumorigenic, irritant, and reproductive effect) were also examined by the Osiris Property Explorer [http://www.organic-chemistry.org/prog/peo].

A diagram of methodological steps used in this work is showed in Figure 1.

\section{Results and Discussion}

Studies of the FLA effects on viruses only have been performed in vitro and in vivo but not in silico using highthroughput (omic) approaches and network analysis based on interactome data. This may occur due to the structure of flavonoids, which generally consist of two aromatic rings, each containing at least one hydroxyl group that is connected through a three-carbon "bridge" becoming later part of a heterocyclic ring [47]. These chemical proprieties allow increased permeability across the cellular membrane to interact with multiple intracellular targets $[48,49]$. As such, these compounds possess a broad spectrum of biological activities [50,51], leading to the overrepresentation of many biological pathways, which may not be necessarily linked to antiviral potential. In this sense, systems pharmacology or chemobiology strategies could be employed to define specific targets of flavonoids.

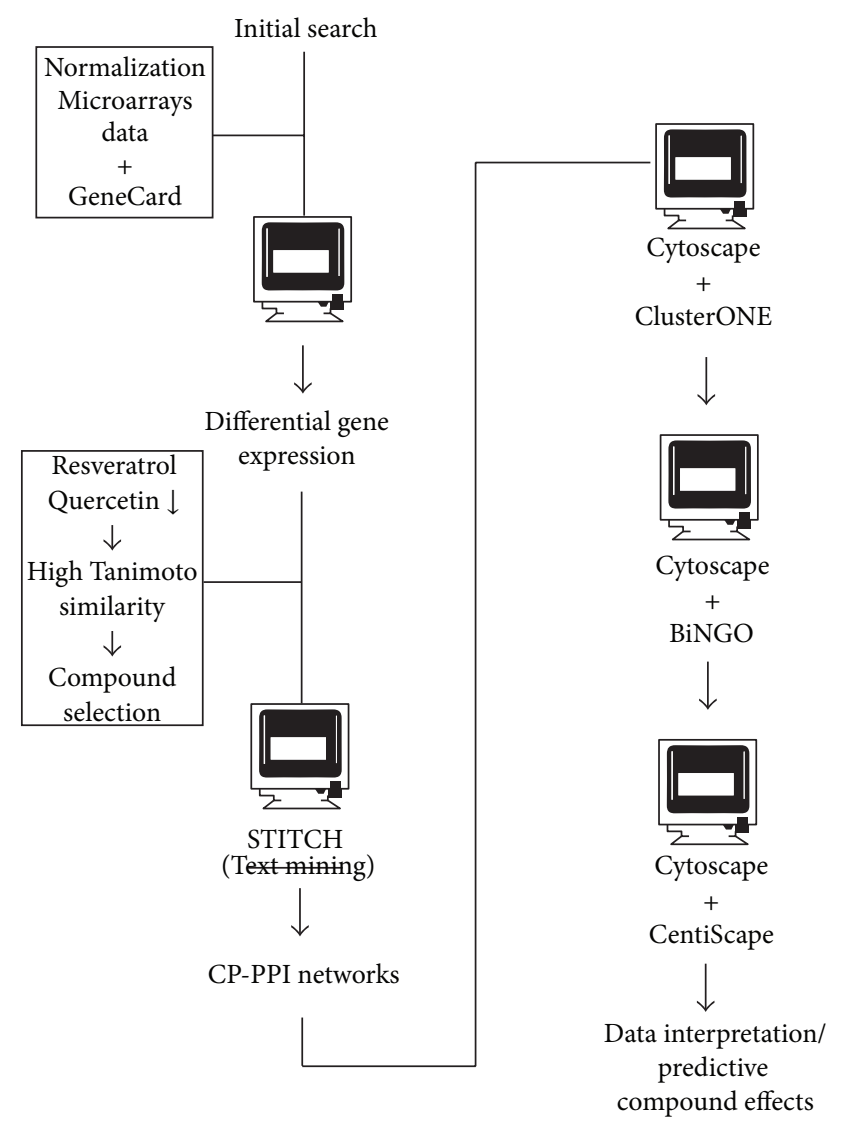

FIGURE 1: Experimental approach employed to define potential treatments against RSV infection. The interactome data was obtained from microarrays data derived from human bronchial cells infected with RSV. Differential gene expression was considered as initial input for network prospection. Additionally, the natural compounds from flavonoids obtained according to Tanimoto similarity were added to the initial input in STITCH software. The CP-PPI network generated was viewed by Cytoscape and analyzed by ClusterONE in order to identify the major clusters associated. Biological processes found within clusters were retrieved by employing BiNGO plugin. Moreover, to find bottlenecks and hubs, proteins/compounds used CentiScape plugin. Finally, data interpretation was performed based on Zinc database and Osiris Property Explorer.

3.1. Topological Design and Analysis of a Major CP-PPI Network of PHBE Cells Infected by RSV. To focus on RSV antiviral effects of flavonoids, we developed an interatomic network considering 285 genes differentially expressed during RSV infection of PHBE cells and 26 flavonoids compounds (Table 1) as an initial input on STITCH software. As a result of this approach, a major CP-PPI network composed of 57 nodes and 92 edges and integrated by five compound targets with putative antiviral activity was obtained (Figure 2). It is important to note that minor networks without CPI were also detected but were not considered for posterior analysis (Supplementary Figure 1; see Supplementary Material available online at http://dx.doi.org/10.1155/2014/301635).

Network topological features could successfully predict FLA mechanisms of action against RSV infection. In this 


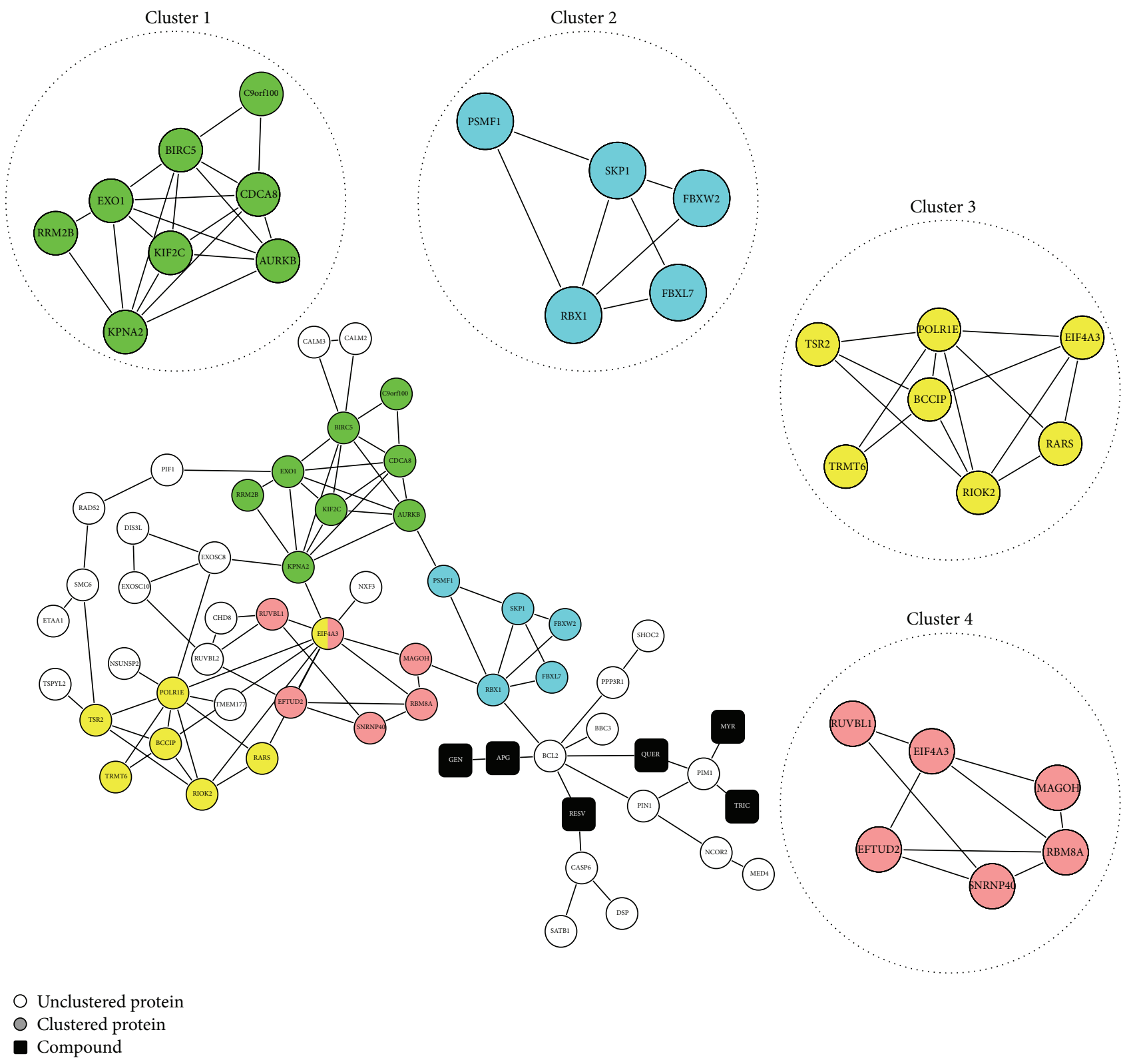

FIGURE 2: ClusterONE analysis of major chemical-protein (CP) and protein-protein interacting (PPI) network. All clustered proteins (composing different subnetworks) and unclustered proteins are represented by nodes of different colors. Chemical compounds are represented by square shape nodes. FLA compounds abbreviations: resveratrol (RESV), apigenin (APG), quercetin (QUER), myricetin (MYR), tricetin (TRIC), and genistein (GEN).

sense, the global organization of clustering in the major network suitable for flavonoid modulation was analyzed. ClusterONE identified four interconnected clusters (Figure 2). Subnetworks of these clusters were created, representing four discrete biological processes, as identified by gene ontology analysis (GO) (Supplementary Table 1): (1) cell cycle phase (corrected $P$ value: $2.33 \times 10^{-6}$ ); (2) ubiquitin-dependent protein catabolic process (corrected $P$ value: $1.61 \times 10^{-5}$ ); (3) nucleic acid metabolic process (corrected $P$ value: $4.68 \times$ $10^{-4}$ ); and (4) RNA splicing (corrected $P$ value: $1.65 \times 10^{-6}$ ). RSV-host studies have identified these processes that occur upon infection [52-54]. However, all flavonoids and their targets are unclustered in the major CP-PPI network. This shows a compound-target regulation independent of cluster network organization during early RSV infection. An alternative and possible strategy to understand RVS modulation by flavonoids is to predict the best ranking of compound target (high impact on the network) through network connectivity analysis. In this sense, centrality properties were evaluated; however, $11 \mathrm{H}$-B nodes were identified in the CP-PPI network, represented only by proteins (Figure 3(a), Supplementary Table 2). These same H-B nodes possess high closeness values (Figure 3(b), Supplementary Table 2), suggesting that these nodes may have close communication with others in the 


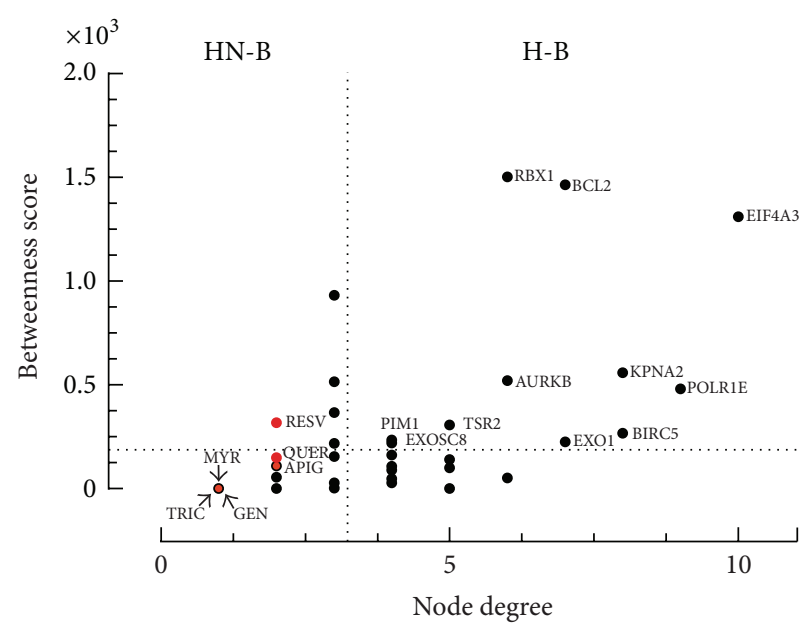

(a)

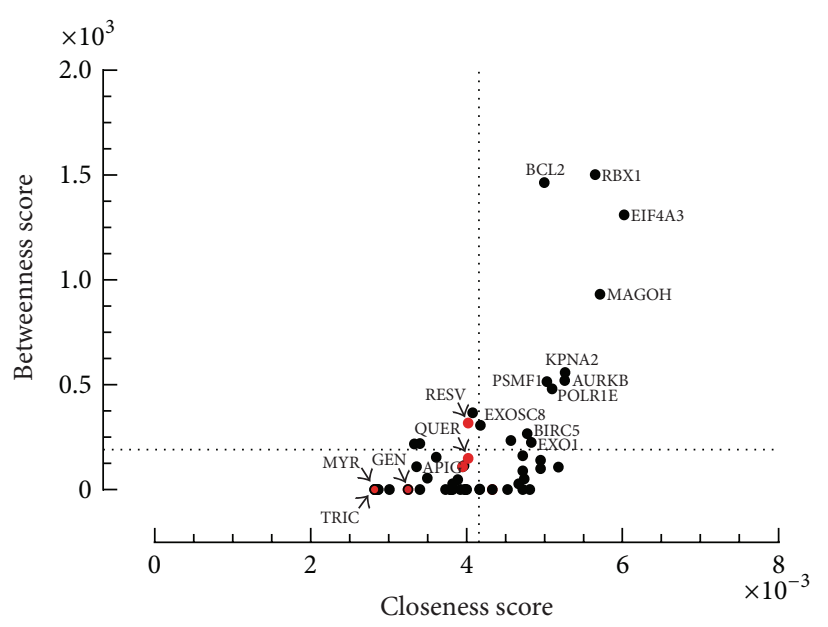

(b)

Figure 3: Centrality analysis ( $\mathrm{a}$ and $\mathrm{b}$ ) of the major CP-PPI network. Dashed lines represent the threshold value calculated for each centrality. Proteins are represented by black dots, while flavonoid compounds are marked in red. Only proteins or flavonoid compounds with bottleneck scores above the network average are indicated. Legend: hub-bottleneck (H-B); non-hub-bottleneck (NH-B). FLA Compounds abbreviations: resveratrol (RESV); apigenin (APG); quercetin (QUER); myricetin (MYR); tricetin (TRIC), and genistein (GEN).

major network. All flavonoid compounds are $\mathrm{H}-\mathrm{NB}$ and $\mathrm{NH}-$ NB nodes, but these modulate directly $2 \mathrm{H}-\mathrm{B}$ proteins (PIM1 and BCL2).

3.1.1. PIM1 and BCL2, as FLA Targets against RSV Infection. PIM1 is a protooncogene which encodes a serine/threonine kinase [55]. This kinase controls cell survival, proliferation, differentiation, and apoptosis [56]. In the context of respiratory diseases, a recent study suggests that PIM1 has a role in the induction of allergic airway responses [57]. Therefore, PIM1 inhibition reduces the development of full spectrum allergen-induced lung inflammatory responses, at least partially through limiting the expansion and actions of CD4+ and CD8 + effector T cells [57]. A similar function for PIM1 has been described in acute RSV infections [58]. PIM1 inhibition attenuates induced RSV reinfection, enhancing airway hyperresponsiveness and activation of the inflammatory cascade. In our analyses, PIM1 showed to be upregulated in comparison with noninfected control $(\log \mathrm{FC}=0.026)$ and to interact with three flavonoids (tricetin, myricetin, and quercetin). These compounds are cell-permeable and directly inhibit PIM1 kinase activity [59]. In this sense, these flavonoids are potential inhibitors of RSV-caused inflammation in a target-specific manner, through yet unknown mechanisms. It is important to note that anti-RSV activity of myricetin and tricetin were not tested experimentally and should be further investigated.

On the other hand, our data suggest BLC2 regulation mediated by flavonoids. BCL2 is a regulator of programmed cell death (apoptosis), in part by modulating the release of proapoptotic molecules from mitochondria. For viruses in general (included RSV), apoptotic death of infected cells is a mechanism for reducing virus replication. After $24 \mathrm{~h}$ of infection by RSV, several proapoptotic factors of the BCL2 family and caspases $3,6,7,8,9$, and 10 are induced in different epithelial cell lines (primary small airway cells, primary tracheal-bronchial cells, A549, and HEp-2 but not for PHBE) [60]. At the same time, RSV also mediates induction of antiapoptotic factors of the BCL2 family [60], which might account for the delayed induction of apoptosis of RSVinfected cells. This indicates the importance of a complex struggle between apoptotic (host) and antiapoptotic (virus) pathways [60].

In our study, BCL2 was shown to be downregulated in PHBE infected cells in comparison with noninfected controls ( $\log \mathrm{FC}=-0.008)$. We hypothesized that differential expression of this gene may be caused by overexpression of PIM1. In hematopoietic cells, PIM1 kinase acts as a survival factor in cooperation with a regulation of BCL2 [61]. This mechanism should be investigated in RSV infected PHBE.

Furthermore, resveratrol and apigenin control the activity of BCL2 in inducing apoptosis in cancer cells $[62,63]$, but the effect of these flavonoids has not been explored in PHBE cells or in in vivo models for RSV infection. However, these compounds are described as inhibitors of RSV replication in vitro (see Table 1).

3.2. In Silico Analysis of FLA Effects on Human Health. We have also predicted potential undesired effects on human health of each of the FLA compounds based on its chemical structures (for more details, see Section 2.7 of Materials and Methods). Our analysis suggests that tricetin may have low risk to human health considering the four main parameters of the analysis (mutagenic, tumorigenic, irritant, and reproductive effectiveness), as shown in Table 2. The other four flavonoids (resveratrol, quercetin, apigenin, and myricetin) may require chemical modification to reduce human health impact but provide versatile chemical backbones for drug development. Biotransformation of flavonoids into drugs is 
TAble 2: Prediction of effects of FLA compounds based on chemical structure.

\begin{tabular}{lcccccccc}
\hline Molecules & $x \log P^{*}$ & H-bond acceptors & H-bond donors & MV $(\mathrm{g} / \mathrm{mol})^{*}$ & $\begin{array}{c}\text { Mutagenic } \\
\text { ** }\end{array}$ & $\begin{array}{c}\text { Tumorigenic } \\
\text { Tut }^{*}\end{array}$ & $\begin{array}{c}\text { Irritant } \\
\text { R* }\end{array}$ & $\begin{array}{c}\text { Reproductive } \\
\text { effect** }\end{array}$ \\
\hline Resveratrol & 2.99 & 3 & 3 & 228.247 & High-risk & Low-risk & Low-risk & High-risk \\
Quercetin & 1.68 & 7 & 5 & 302.238 & High-risk & Medium-risk & Low-risk & Medium-risk \\
Apigenin & 2.46 & 5 & 3 & 270.24 & High-risk & Medium-risk & Low-risk & High-risk \\
Genistein & 2.27 & 5 & 3 & 270.24 & High-risk & High-risk & Low-risk & High-risk \\
Myricetin & 1.39 & 8 & 6 & 318.237 & High-risk & Low-risk & Low-risk & Low-risk \\
Tricetin & 1.68 & 5 & 7 & 302.238 & Low-risk & Low-risk & Low-risk & Low-risk \\
\hline
\end{tabular}

* All parameters related to Lipinsky's rule of five were obtained from Zinc database.

**A All toxicity risks were predicted by Osiris Property Explorer.

the usual approach in the development of anticancer targets $[64,65]$ but could also be applied in the search of new therapies against RSV.

\section{Conclusions}

Our model network CPI-PPI identified five target flavonoid compounds: resveratrol, quercetin, tricetin, apigenin, and myricetin. These compounds are suggested as potential candidates in the process of development of novel drugs against early severe RSV infection. Despite these potentially interesting associations, these findings are mainly relying on statistical analysis. Thus, Further experimental testing of these predictions will be required to support the in silico data.

\section{Conflict of Interests}

The authors declare that there is no conflict of interests regarding the publication of this paper.

\section{Acknowledgments}

This study was supported by Coordenação de Aperfeiçoamento de Pessoal de Nivel Superior (CAPES) Grant no. 02775/09-3. José Eduardo Vargas received postdoctoral fellowships from CAPES-PNPD program.

\section{References}

[1] F. W. Denny and F. A. Loda, "Acute respiratory infections are the leading cause of death in children in developing countries," The American Journal of Tropical Medicine and Hygiene, vol. 35, no. 1, pp. 1-2, 1986.

[2] C. B. Hall, G. A. Weinberg, M. K. Iwane et al., "The Burden of respiratory syncytial virus infection in young children," The New England Journal of Medicine, vol. 360, no. 6, pp. 588-598, 2009.

[3] B. G. Williams, E. Gouws, C. Boschi-Pinto, J. Bryce, and C. Dye, "Estimates of world-wide distribution of child deaths from acute respiratory infections," The Lancet Infectious Diseases, vol. 2, no. 1, pp. 25-32, 2002.

[4] W. P. Glezen, L. H. Taber, A. L. Frank, and J. A. Kasel, "Risk of primary infection and reinfection with respiratory syncytial virus," The American Journal of Diseases of Children, vol. 140, no. 6, pp. 543-546, 1986.
[5] H. W. Kim, J. G. Canchola, C. D. Brandt et al., "Respiratory syncytial virus disease in infants despite prior administration of antigenic inactivated vaccine," American Journal of Epidemiology, vol. 89, no. 4, pp. 422-434, 1969.

[6] J. A. Espinoza, S. M. Bueno, C. A. Riedel, and A. M. Kalergis, "Induction of protective effector immunity to prevent pathogenesis caused by the respiratory syncytial virus. Implications on therapy and vaccine design," Immunology, vol. 143, no. 1, pp. $1-12,2014$.

[7] R. A. Karron, B. Thumar, E. Schappell, U. J. Buchholz, and P. L. Collins, "Attenuation of live respiratory syncytial virus vaccines is associated with reductions in levels of nasal cytokines," The Journal of Infectious Diseases, vol. 207, no. 11, pp. 1773-1779, 2013.

[8] R. J. Loomis and P. R. Johnson, "Gene-based vaccine approaches for respiratory syncytial virus," Current Topics in Microbiology and Immunology, vol. 372, pp. 307-324, 2013.

[9] T. G. Morrison and E. E. Walsh, "Subunit and virus-like particle vaccine approaches for respiratory syncytial virus," Current Topics in Microbiology and Immunology, vol. 372, pp. 285-306, 2013.

[10] "Palivizumab, a humanized respiratory syncytial virus monoclonal antibody, reduces hospitalization from respiratory syncytial virus infection in high-risk infants," Pediatrics, vol. 102, no. 3, pp. 531-537, 1998.

[11] W. A. Prescott Jr., F. Doloresco, J. Brown, and J. A. Paladino, "Cost effectiveness of respiratory syncytial virus prophylaxis: a critical and systematic review," PharmacoEconomics, vol. 28, no. 4, pp. 279-293, 2010.

[12] P. G. Canonico, M. D. Kastello, C. T. Spears, J. R. Brown, E. A. Jackson, and D. E. Jenkins, "Effects of ribavirin on red blood cells," Toxicology and Applied Pharmacology, vol. 74, no. 2, pp. 155-162, 1984.

[13] G. Oswald, K. Alzoubi, M. Abed, and F. Lang, "Stimulation of suicidal erythrocyte death by ribavirin," Basic \& Clinical Pharmacology \& Toxicology, vol. 114, no. 4, pp. 311-317, 2013.

[14] A. Cantatore, S. D. Randall, D. Traum, and S. D. Adams, "Effect of black tea extract on herpes simplex virus-1 infection of cultured cells," BMC Complementary and Alternative Medicine, vol. 13, article 139, 2013.

[15] J. M. Davis, E. A. Murphy, J. L. McClellan, M. D. Carmichael, and J. D. Gangemi, "Quercetin reduces susceptibility to influenza infection following stressful exercise," American Journal of Physiology-Regulatory Integrative and Comparative Physiology, vol. 295, no. 2, pp. R505-R509, 2008.

[16] B. Sritularak, K. Tantrakarnsakul, V. Lipipun, and K. Likhitwitayawuid, "Flavonoids with anti-HSV activity from 
the root bark of Artocarpus lakoocha," Natural Product Communications, vol. 8, no. 8, pp. 1079-1080, 2013.

[17] G. Smyth, "Limma: linear models for microarray data," in Bioinformatics and Computational Biology Solutions using R and Bioconductor, pp. 397-420, Springer, Berlin, Germany, 2005.

[18] R. Breitling, P. Armengaud, A. Amtmann, and P. Herzyk, "Rank products: a simple, yet powerful, new method to detect differentially regulated genes in replicated microarray experiments," FEBS Letters, vol. 573, no. 1-3, pp. 83-92, 2004.

[19] T. N. Kaul, E. Middleton Jr., and P. L. Ogra, "Antiviral effect of flavonoids on human viruses," Journal of Medical Virology, vol. 15, no. 1, pp. 71-79, 1985.

[20] Y. H. Kim, C. Y. Choi, and Y. Kim, "Covalent modification of the homeodomain-interacting protein kinase 2 (HIPK2) by the ubiquitin-like protein SUMO-1," Proceedings of the National Academy of Sciences of the United States of America, vol. 96, no. 22, pp. 12350-12355, 1999.

[21] M. Thapa, Y. Kim, J. Desper, K.-O. Chang, and D. H. Hua, "Synthesis and antiviral activity of substituted quercetins," Bioorganic and Medicinal Chemistry Letters, vol. 22, no. 1, pp. 353-356, 2012.

[22] L. Drago, L. Nicola, F. Ossola, and E. de Vecchi, "In vitro antiviral activity of resveratrol against respiratory viruses," Journal of Chemotherapy, vol. 20, no. 3, pp. 393-394, 2008.

[23] T. Liu, N. Zang, N. Zhou et al., "Resveratrol inhibits the TRIFdependent pathway by upregulating sterile alpha and armadillo motif protein, contributing to anti-inflammatory effects after respiratory syncytial virus infection," Journal of Virology, vol. 88, no. 8, pp. 4229-4236, 2014.

[24] X. H. Xie, N. Zang, S. M. Li et al., "Resveratrol Inhibits respiratory syncytial virus-induced IL-6 production, decreases viral replication, and downregulates TRIF expression in airway epithelial cells," Inflammation, vol. 35, no. 4, pp. 1392-1401, 2012.

[25] R. J. Nijveldt, E. van Nood, D. E. C. van Hoorn, P. G. Boelens, K. van Norren, and P. A. M. van Leeuwen, "Flavonoids: a review of probable mechanisms of action and potential applications," The American Journal of Clinical Nutrition, vol. 74, no. 4, pp. 418425, 2001.

[26] R. P. Garofalo, D. Kolli, and A. Casola, "Respiratory syncytial virus infection: mechanisms of redox control and novel therapeutic opportunities," Antioxidants and Redox Signaling, vol. 18, no. 2, pp. 186-217, 2013.

[27] W. D. Guan, Z. F. Yang, N. Liu, S. Qin, F. X. Zhang, and Y. T. $\mathrm{Zhu}$, "[In vitro experimental study on the effect of resveratrol against several kinds of respiroviruses]," Zhong Yao Cai, vol. 31, no. 9, pp. 1388-1390, 2008.

[28] J. Li, S. Wang, J. Xu et al., "Regulation trend of resveratrol on TNF- $\alpha$, IL-1 $\beta$, IL- 6 expressions in bronchoalveolar lavage fluid of RSV-infected BALB/c mice," Zhongguo Zhong Yao Za Zhi, vol. 37, no. 10, pp. 1451-1454, 2012.

[29] N. Zang, X. Xie, Y. Deng et al., "Resveratrol-mediated gamma interferon reduction prevents airway inflammation and airway hyperresponsiveness in respiratory syncytial virus-infected immunocompromised mice," Journal of Virology, vol. 85, no. 24, pp. 13061-13068, 2011.

[30] J. L. Douglas, M. L. Panis, E. Ho et al., "Inhibition of respiratory syncytial virus fusion by the small molecule VP-14637 via specific interactions with F protein," Journal of Virology, vol. 77, no. 9, pp. 5054-5064, 2003.

[31] D. Lai, D. C. Odimegwu, C. Esimone, T. Grunwald, and P. Proksch, "Phenolic compounds with in vitro activity against respiratory syncytial virus from the nigerian lichen Ramalina farinacea," Planta Medica, vol. 79, no. 15, pp. 1440-1446, 2013.

[32] S. Sakai, H. Kawamata, T. Kogure et al., "Inhibitory effect of ferulic acid and isoferulic acid on the production of macrophage inflammatory protein-2 in response to respiratory syncytial virus infection in RAW264.7 cells," Mediators of Inflammation, vol. 8, no. 3, pp. 173-175, 1999.

[33] Y. Wang, M. Chen, J. Zhang et al., "Flavone C-glycosides from the leaves of Lophatherum gracile and their in vitro antiviral activity," Planta Medica, vol. 78, no. 1, pp. 46-51, 2012.

[34] S.-C. Ma, J. Du, P. P.-H. But et al., "Antiviral Chinese medicinal herbs against respiratory syncytial virus," Journal of Ethnopharmacology, vol. 79, no. 2, pp. 205-211, 2002.

[35] D. L. Barnard, J. H. Huffman, L. R. Meyerson, and R. W. Sidwell, "Mode of inhibition of respiratory syncytial virus by a plant flavonoid, SP-303," Chemotherapy, vol. 39, no. 3, pp. 212-217, 1993.

[36] H. W. M. Rixon, G. Brown, J. T. Murray, and R. J. Sugrue, "The respiratory syncytial virus small hydrophobic protein is phosphorylated via a mitogen-activated protein kinase p38dependent tyrosine kinase activity during virus infection," Journal of General Virology, vol. 86, no. 2, pp. 375-384, 2005.

[37] P. Willett, J. M. Barnard, and G. M. Downs, "Chemical similarity searching," Journal of Chemical Information and Computer Sciences, vol. 38, no. 6, pp. 983-996, 1998.

[38] P. Shannon, A. Markiel, O. Ozier et al., "Cytoscape: a software Environment for integrated models of biomolecular interaction networks," Genome Research, vol. 13, no. 11, pp. 2498-2504, 2003.

[39] T. Nepusz, H. Yu, and A. Paccanaro, "Detecting overlapping protein complexes in protein-protein interaction networks," Nature Methods, vol. 9, no. 5, pp. 471-472, 2012.

[40] S. Maere, K. Heymans, and M. Kuiper, "BiNGO: a cytoscape plugin to assess overrepresentation of gene ontology categories in biological networks," Bioinformatics, vol. 21, no. 16, pp. 34483449, 2005.

[41] I. Rivals, L. Personnaz, L. Taing, and M. C. Potier, "Enrichment or depletion of a GO category within a class of genes: which test?" Bioinformatics, vol. 23, no. 4, pp. 401-407, 2007.

[42] Y. Benjamini, D. Drai, G. Elmer, N. Kafkafi, and I. Golani, “Controlling the false discovery rate in behavior genetics research," Behavioural Brain Research, vol. 125, no. 1-2, pp. 279-284, 2001.

[43] G. Scardoni, M. Petterlini, and C. Laudanna, "Analyzing biological network parameters with CentiScaPe," Bioinformatics, vol. 25, no. 21, pp. 2857-2859, 2009.

[44] M. E. J. Newman, "A measure of betweenness centrality based on random walks," Social Networks, vol. 27, no. 1, pp. 39-54, 2005.

[45] J. J. Irwin, T. Sterling, M. M. Mysinger, E. S. Bolstad, and R. G. Coleman, "ZINC: a free tool to discover chemistry for biology," Journal of Chemical Information and Modeling, vol. 52, no. 7, pp. 1757-1768, 2012.

[46] J. J. Irwin and B. K. Shoichet, "ZINC-a free database of commercially available compounds for virtual screening," Journal of Chemical Information and Modeling, vol. 45, no. 1, pp. 177-182, 2005.

[47] S. J. Flora, "Structural, chemical and biological aspects of antioxidants for strategies against metal and metalloid exposure," Oxidative Medicine and Cellular Longevity, vol. 2, no. 4, pp. 191206, 2009. 
[48] J. Brittes, M. Lúcio, C. Nunes, J. L. F. C. Lima, and S. Reis, "Effects of resveratrol on membrane biophysical properties: relevance for its pharmacological effects," Chemistry and Physics of Lipids, vol. 163, no. 8, pp. 747-754, 2010.

[49] S. Chaudhuri, A. Banerjee, K. Basu, B. Sengupta, and P. K. Sengupta, "Interaction of flavonoids with red blood cell membrane lipids and proteins: antioxidant and antihemolytic effects," International Journal of Biological Macromolecules, vol. 41, no. 1, pp. 42-48, 2007.

[50] A. B. Hendrich, "Flavonoid-membrane interactions: possible consequences for biological effects of some polyphenolic compounds," Acta Pharmacologica Sinica, vol. 27, no. 1, pp. 27-40, 2006.

[51] E. Middleton Jr., C. Kandaswami, and T. C. Theoharides, "The effects of plant flavonoids on mammalian cells: implications for inflammation, heart disease, and cancer," Pharmacological Reviews, vol. 52, no. 4, pp. 673-751, 2000.

[52] A. Bakre, P. Mitchell, J. K. Coleman et al., "Respiratory syncytial virus modifies microRNAs regulating host genes that affect virus replication," Journal of General Virology, vol. 93, part 11, pp. 2346-2356, 2012.

[53] J. Elliott, O. T. Lynch, Y. Suessmuth et al., "Respiratory syncytial virus NS1 protein degrades STAT2 by using the elongin-cullin E3 ligase," Journal of Virology, vol. 81, no. 7, pp. 3428-3436, 2007.

[54] W. Wu, D. C. Munday, G. Howell, G. Platt, J. N. Barr, and J. A. Hiscox, "Characterization of the interaction between human respiratory syncytial virus and the cell cycle in continuous cell culture and primary human airway epithelial cells," Journal of Virology, vol. 85, no. 19, pp. 10300-10309, 2011.

[55] M. Bachmann and T. Möröy, "The serine/threonine kinase Pim1," International Journal of Biochemistry and Cell Biology, vol. 37, no. 4, pp. 726-730, 2005.

[56] Z. Wang, N. Bhattacharya, M. Weaver et al., "Pim-1: a serine/threonine kinase with a role in cell survival, proliferation, differentiation and tumorigenesis," Journal of Veterinary Science, vol. 2, no. 3, pp. 167-179, 2001.

[57] Y. S. Shin, K. Takeda, Y. Shiraishi et al., "Inhibition of Pim1 kinase activation attenuates allergen-induced airway hyperresponsiveness and inflammation," The American Journal of Respiratory Cell and Molecular Biology, vol. 46, no. 4, pp. 488497, 2012.

[58] J. Han, W. Zeng, M. Wang et al., "Inhibition of Piml kinase attenuates respiratory syncytial virus (RSV) re-infectioninduced enhanced airway hyperresponsiveness (AHR) and inflammation," The Journal of Allergy and Clinical Immunology, vol. 131, no. 2, p. AB75, 2013.

[59] S. Holder, M. Lilly, and M. L. Brown, "Comparative molecular field analysis of flavonoid inhibitors of the PIM-1 kinase," Bioorganic and Medicinal Chemistry, vol. 15, no. 19, pp. 64636473, 2007.

[60] A. Kotelkin, E. A. Prikhod'ko, J. I. Cohen, P. L. Collins, and A. Bukreyev, "Respiratory syncytial virus infection sensitizes cells to apoptosis mediated by tumor necrosis factor-related apoptosis-inducing ligand," Journal of Virology, vol. 77, no. 17, pp. 9156-9172, 2003.

[61] M. Lilly, J. Sandholm, J. J. Cooper, P. J. Koskinen, and A. Kraft, "The PIM-1 serine kinase prolongs survival and inhibits apoptosis-related mitochondrial dysfunction in part through a bcl-2-dependent pathway," Oncogene, vol. 18, no. 27, pp. 40224031, 1999.

[62] S. Ganapathy, Q. Chen, K. P. Singh, S. Shankar, and R. K. Srivastava, "Resveratrol enhances antitumor activity of TRAIL in prostate cancer xenografts through activation of FOXO transcription factor," PLoS ONE, vol. 5, no. 12, Article ID e15627, 2010.

[63] Y. Zhu, Y. Mao, H. Chen et al., "Apigenin promotes apoptosis, inhibits invasion and induces cell cycle arrest of T24 human bladder cancer cells," Cancer Cell International, vol. 13, no. 1, article 54, 2013.

[64] A. Bartmańska, T. Tronina, J. Popłoński, and E. Huszcza, "Biotransformations of prenylated hop flavonoids for drug discovery and production," Current Drug Metabolism, vol. 14, no. 10, pp. 1083-1097, 2013.

[65] M. K. Chahar, N. Sharma, M. P. Dobhal, and Y. C. Joshi, "Flavonoids: a versatile source of anticancer drugs," Pharmacognosy Reviews, vol. 5, no. 9, pp. 1-12, 2011. 

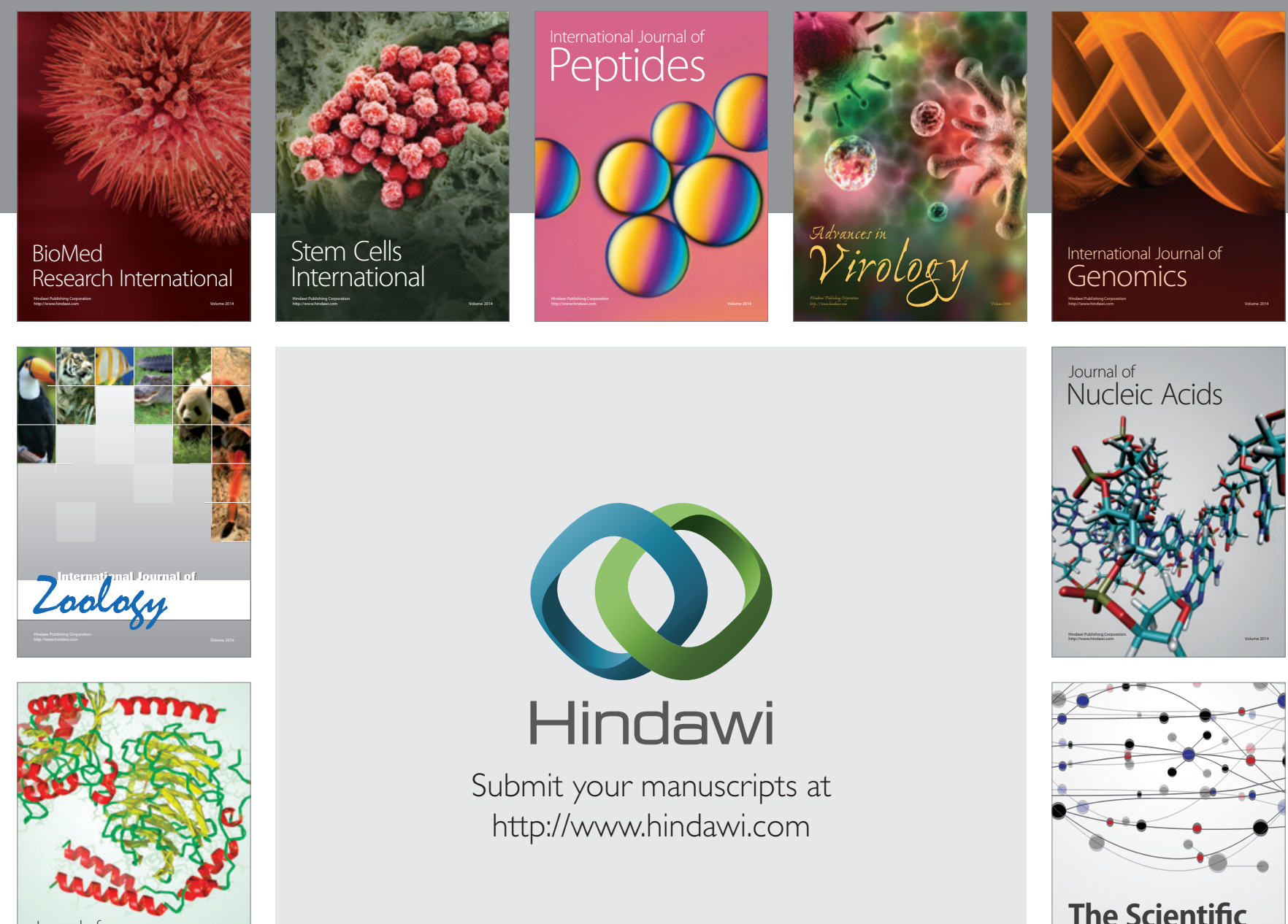

Submit your manuscripts at

http://www.hindawi.com

Journal of
Signal Transduction
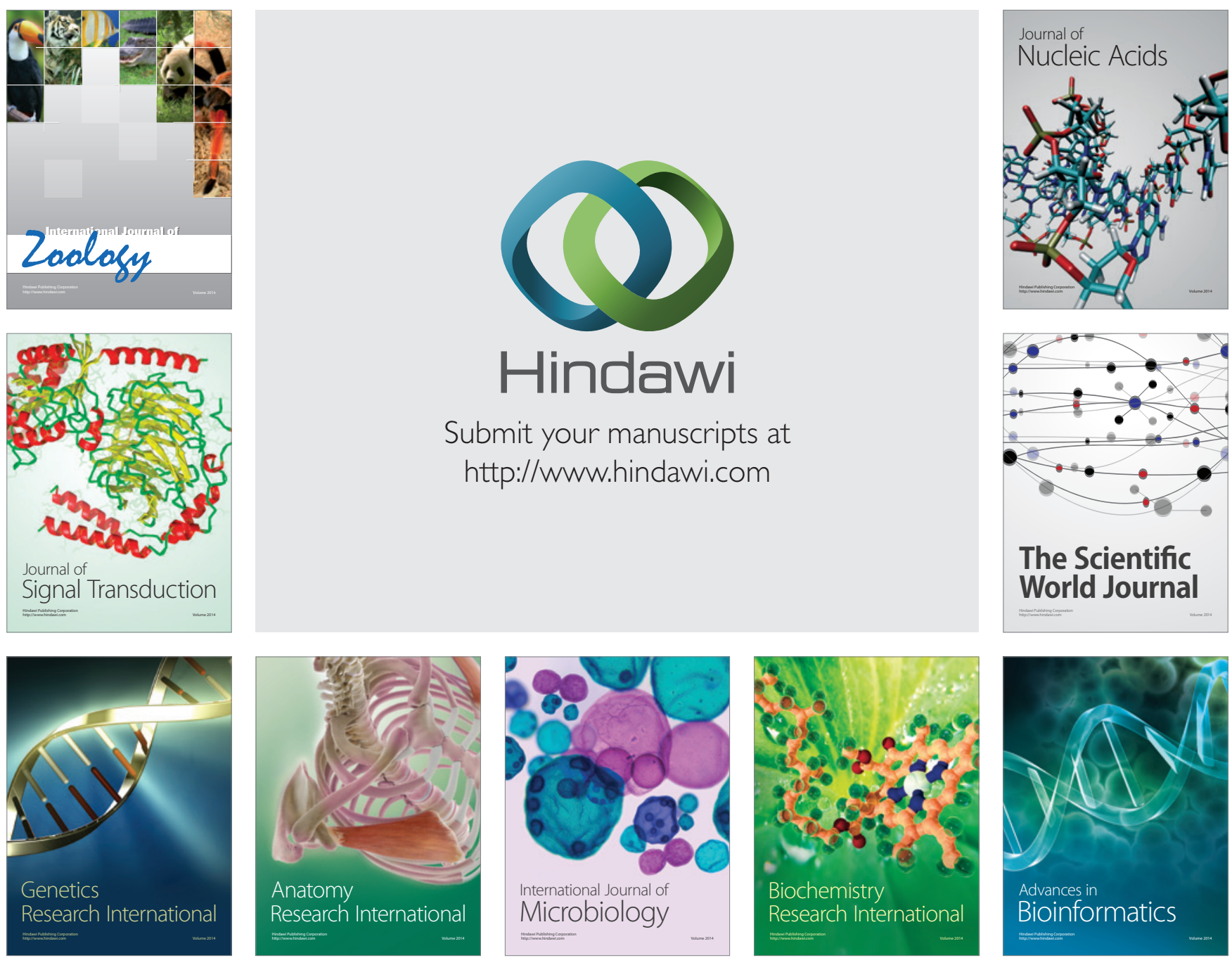

The Scientific World Journal
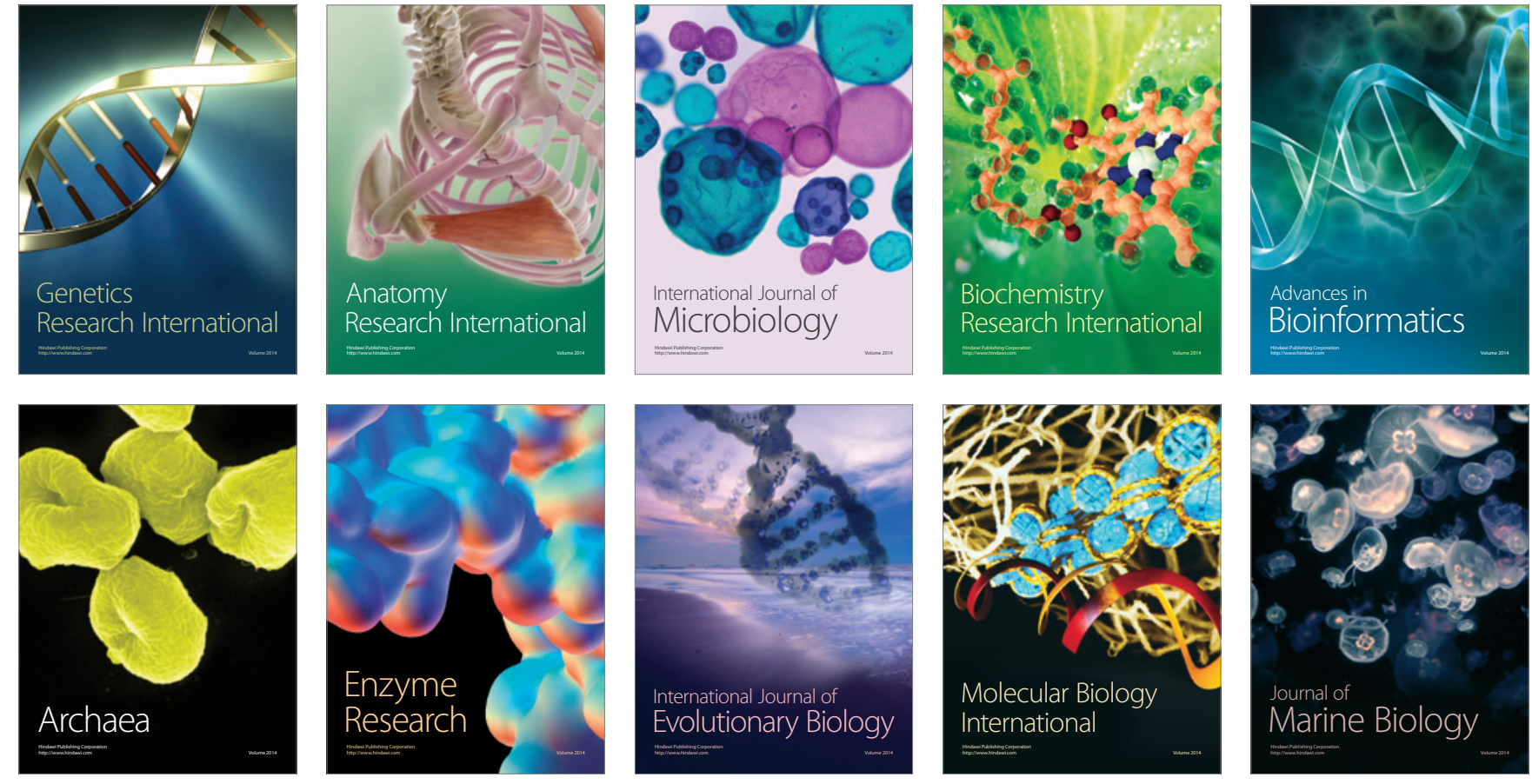